Efficiency droop in InGaN/GaN multiple quantum well light-emitting diodes with nonuniform current spreading

This article has been downloaded from IOPscience. Please scroll down to see the full text article.

2011 Semicond. Sci. Technol. 26095007

(http://iopscience.iop.org/0268-1242/26/9/095007)

View the table of contents for this issue, or go to the journal homepage for more

Download details:

IP Address: 91.209.11.94

The article was downloaded on 08/07/2011 at 10:29

Please note that terms and conditions apply. 


\title{
Efficiency droop in InGaN/GaN multiple quantum well light-emitting diodes with nonuniform current spreading
}

\author{
Ya Ya Kudryk ${ }^{1}$ and A V Zinovchuk ${ }^{2}$ \\ ${ }^{1}$ V. Lashkaryov Institute of Semiconductor Physics, 03028 Kyiv, Ukraine \\ ${ }^{2}$ Ivan Franko Zhytomyr State University, 10008 Zhytomyr, Ukraine \\ E-mail: zinovchuk.a@zu.edu.ua
}

Received 19 April 2011, in final form 9 June 2011

Published 6 July 2011

Online at stacks.iop.org/SST/26/095007

\begin{abstract}
We demonstrate that the efficiency droop phenomenon in multiple quantum well InGaN/GaN light-emitting diodes (LEDs) may be connected to the current crowding effect. A numerical model of internal quantum efficiency calculation is presented that takes into account nonuniform lateral carrier injection in the active region. Based on this model, we examine the effect of current crowding on the efficiency droop using comparison of simulated internal quantum efficiency of InGaN LEDs with low and high uniformity of current spreading. The results of simulations and measurements show that the devices with low uniformity of current spreading exhibit higher efficiency droop and lower roll-off current value.
\end{abstract}

\section{Introduction}

InGaN/GaN multiple quantum well (MQW) light-emitting diodes (LEDs) have attracted much attention because of their applications in general illuminations, back lighting and displays. The research in this field has led to great progress in material quality, efficiency and lumen output of nitridebased LEDs. Despite this, the external quantum efficiency (EQE) of MQW InGaN/GaN LEDs reaches its peak at low current density (typically $<20 \mathrm{~A} \mathrm{~cm}^{-2}$ ) and gradually decreases with further increase of current (the so-called 'efficiency droop' phenomenon). This nonthermal efficiency roll-off is observed in ultraviolet, blue and green nitridebased LEDs. Various explanations of physical mechanisms leading to efficiency droop have been proposed in recent years. Auger recombination $[1,2]$ and carrier delocalization from In-rich regions [3, 4] are such mechanisms related to the recombination in quantum wells. Poor hole transport through the quantum barriers [5] and piezoelectric polarization fields $[6,7]$ that cause electron overflow to the p-type region are transport-based (leakage processes) explanations of the efficiency droop. Inefficient carrier injection [8] and the effect of electron blocking layer [9] also have been proposed as the transport-based mechanisms. To date, however, the origin of the droop is not fully understood and is still under debate.

A major part of commercially available nitride-based LEDs employ lateral carrier injection geometry and thus suffer from the current crowding (CC) effect. This effect is due to localization of the current flow routes in some regions of a multilayer structure. It is well known in InGaN/GaN LEDs grown on insulating sapphire substrates [10, 11] and becomes an important problem for large-area high-power devices. In this work, we investigated the effect of $\mathrm{CC}$ on the efficiency droop in lateral InGaN/GaN LEDs. A theoretical model is proposed that includes a $3 \mathrm{D}$ numerical simulation of current density distributions in an LED die and internal quantum efficiency (IQE) calculations by means of recombination coefficient $(A B C)$ equation. Contrary to the previously reported calculations, this two-stage model allows us to simulate the IQE versus current dependences with respect to nonuniform lateral carrier injection in the active region. In addition, we present experimental results on the EQE versus current dependences in blue and green InGaN/GaN LEDs with two different contact electrode geometries. The values of efficiency droop of these LEDs are compared and analysed in terms of the proposed model. 


\section{Simulation and experiment}

In order to theoretically investigate the effect of $\mathrm{CC}$ on the efficiency of InGaN LEDs, we propose a two-stage model. The first stage includes the calculation of current density distributions in an LED die at different applied biases. For the numerical 3D simulation of current spreading, we consider the conventional bar-shaped contact geometry of InGaN LEDs on a sapphire substrate (figure 1) with commonly accepted material parameters and dimensions: $n$-GaN layer $\left(d_{n}=\right.$ $3 \mu \mathrm{m}, n=5 \times 10^{18} \mathrm{~cm}^{-3}$ ), active layer with nonlinear $\mathrm{p}-\mathrm{n}$ junction conductivity, $p$-GaN layer $\left(d_{p}=0.1 \mu \mathrm{m}\right.$, $\left.p=5 \times 10^{17} \mathrm{~cm}^{-3}\right), 1 \times 1 \mathrm{~mm}^{2}$ area. The simulation of the current flow was performed with the finite-element simulation. It was assumed that the electric charges are localized in the space-charge region of the $\mathrm{p}-\mathrm{n}$ junction and the other regions of the structure are neutral. Furthermore, the diffusion component of the current in these regions may be neglected. Therefore, the electric potential distribution follows from the equation $\vec{\nabla}\left(\sigma\left(x, y, z, V_{\text {active }}\right) \vec{\nabla} \varphi\right)=0$. The current density is connected to the potential via $\vec{J}=-\sigma\left(x, y, z, V_{\text {active }}\right) \vec{\nabla} \varphi$ (where $\sigma\left(x, y, z, V_{\text {active }}\right)$ is the conductivity of the layers and $V_{\text {active }}$ is the voltage drop across the active layer). The conductivity of the active layer is represented by using the diode-like current-voltage dependence $J=J_{S}\left(\exp \left(e V_{\text {active }} / \beta k T\right)-1\right)$, where $J$ is the vertical current density locally controlled by the $V_{\text {active }}$ value, $J_{s}$ is the saturation current density, $\beta$ is the $\mathrm{p}-\mathrm{n}$ junction ideality factor, $e$ is the elementary charge, $k$ is Boltzmann's constant and $T$ is the temperature. We suppose that $\beta=2$ (recombination process in the space-charge region) and the saturation current $J_{s}=1.5 \times 10^{-20} \mathrm{~A} \mathrm{~cm}^{-2}$. The mobilities of electrons and holes are assumed to be $\mu_{n}=600 \mathrm{~cm}^{2} \mathrm{~V}^{-1} \mathrm{~s}^{-1}$ and $\mu_{p}=20 \mathrm{~cm}^{2} \mathrm{~V}^{-1} \mathrm{~s}^{-1}$, respectively. The contact resistance and resistance of metal-GaN junction were not taken into account. Such a simple model does not take into account either tunnelling or carrier leakage processes. This allows us to eliminate any transport-based mechanisms from the theoretical analysis of efficiency roll-off behaviour at high currents.

As soon as the current density distributions are calculated, the IQE as a function of the current can be computed (the second stage). The IQE inside the MQW active region is analysed by means of the $A B C$ model. According to it, the IQE can be expressed as follows:

$$
\eta=\frac{B n^{2}}{A n+B n^{2}+C n^{3}},
$$

where $A, B$ and $C$ are the Shockley-Read-Hall (SRH), radiative and Auger coefficients, and $n$ is the injected carrier concentration.

Taking into account the CC-induced nonuniform lateral carrier injection in the active region, it is apparent that the $\eta$ value becomes spatially dependent. Thus equation (1) may be interpreted as the local IQE. In other words, the optical power $(\mathrm{d} P)$ emitted by the elementary area $(\mathrm{d} S)$ of the active region is locally controlled by the $\eta$ value. As is commonly accepted, the total IQE is defined as a ratio of total number of photons emitted from the active region per second to the number of electrons injected into LED per second [12]. The total number

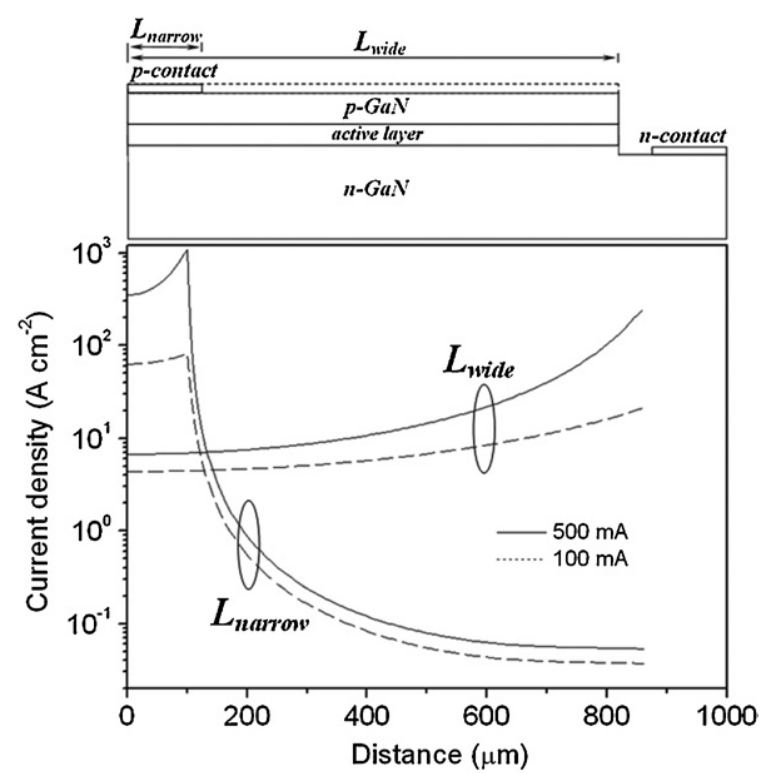

Figure 1. Cross-section of the lateral LED structure used in simulation and current density distributions in the active layer of LEDs with different p-contact widths at $I=100$ and $500 \mathrm{~mA}$.

of emitted photons may be determined by surface integration of the $\mathrm{d} P$ value over the whole active area:

$$
\frac{P}{h v}=\frac{1}{e} \iint \eta(x, y) J(x, y) \mathrm{d} S .
$$

Using equation (2), the total IQE can be presented as

$$
\eta_{\text {tot }}=\frac{\iint \eta(x, y) J(x, y) \mathrm{d} S}{\iint J(x, y) \mathrm{d} S} .
$$

Under the steady-state condition, the relationship between the current density and the injected carrier density is given by

$$
\frac{J}{e d_{\text {active }}}=A n+B n^{2}+C n^{3}
$$

where $d_{\text {active }}$ is the active region thickness.

By using the function $J(x, y)$ obtained at the first stage of the modelling, one can numerically calculate the injected carrier density distribution and function $\eta(x, y)$ from equations (4) and (1). Finally, the total IQE can be obtained by numerical integration in equation (3).

The experimental studies are performed on commercially available large-area $\left(1 \times 1 \mathrm{~mm}^{2}\right)$ blue and green $\mathrm{InGaN} / \mathrm{GaN}$ MQW LEDs grown on a sapphire substrate using the metal organic chemical vapour deposition. The room temperature peak wavelengths of the LEDs at $10 \mathrm{~mA}$ were $\lambda \sim$ $460 \mathrm{~nm}$ (blue) and $\lambda \sim 520 \mathrm{~nm}$ (green). Two different electrode patterns of p-side up mesastructure are investigated: conventional bar-shaped and interdigitated contact pattern (figure 3). All LEDs had the same internal structure consisting of an $n$-GaN layer, a MQW active region comprising five $2 \mathrm{~nm}$ thick InGaN wells and 12-nm thick GaN barriers, an AlGaN electron blocking layer and a $p$-GaN layer. The In content in InGaN wells was approximately $\sim 0.15$ and 0.3 for blue and green LEDs, respectively.

The measurements of the light-current and the currentvoltage characteristics are performed in the pulsed mode, with 


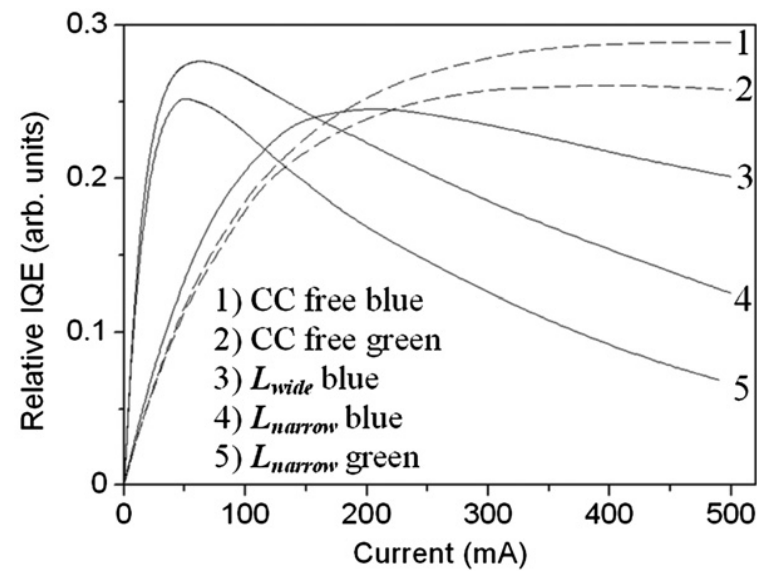

Figure 2. Calculated IQE versus current dependences for blue $(1,3,4)$ and green $(2,5)$ LEDs with a different width of the p-contact.

$1 \%$ duty cycle and $500 \mathrm{~Hz}$ frequency, in order to eliminate the self-heating effect. The spatial electroluminescence patterns are monitored with the optical microscope connected to the CCD camera.

\section{Results and discussion}

The specific features of current spreading in lateral InGaN LEDs were analysed by considering two structures with different widths of the p-contact: $L_{\text {wide }}=850 \mu \mathrm{m}$ (that is equal to the $p$-GaN layer width) and $L_{\text {narrow }}=100 \mu \mathrm{m}$ (figure 1 ). In the LED with wide p-contact $\left(L_{\text {wide }}\right)$, the current density distribution remains practically uniform over the whole active layer up to $I=100 \mathrm{~mA}$. At higher currents, there is a gradual increase of current density at the edge of the p-contact as compared to the average current density. At $I=500 \mathrm{~mA}$ (maximal current value used in simulation), the ratio of peakto-average current density is $\sim 80$. Contrary to that, in the LED with narrow p-contact $\left(L_{\text {narrow }}\right)$, an increase of the applied bias is accompanied by essential localization of the current lines under the p-contact, even at low driving currents. The peakto-average ratio of the current density reaches a value of $10^{2}$ at $I=5 \mathrm{~mA}$, while at $I=500 \mathrm{~mA}$, it goes as high as $2 \times 10^{4}$.

The current density distributions obtained at different applied biases were used to determine the IQE of both LEDs. To this end, we made some assumptions. The thickness of the active layer was assumed to be $10 \mathrm{~nm}$, i.e. the total thickness of five quantum wells. The SRH recombination coefficient $(A)$ directly depends on the substrate used and on the quality of the growing process. For most industrial blue LEDs grown on a sapphire substrate, SRH coefficients are in the range $6.0 \times 10^{7}-2.0 \times 10^{8} \mathrm{~s}^{-1}$ [13]. In this study, we employ the value $2.0 \times 10^{8} \mathrm{~s}^{-1}$. For the radiative recombination coefficient $(B)$ and Auger coefficient $(C)$, we use the values $2.0 \times 10^{-11} \mathrm{~cm}^{3} \mathrm{~s}^{-1}$ and $2.0 \times 10^{-30} \mathrm{~cm}^{6} \mathrm{~s}^{-1}$ obtained from the photoluminescence measurements [14]. Similar values are also obtained from the differential carrier lifetime measurements [15].

Figure 2 presents the calculated IQE as a function of current for blue LEDs with a different width of the p-contact

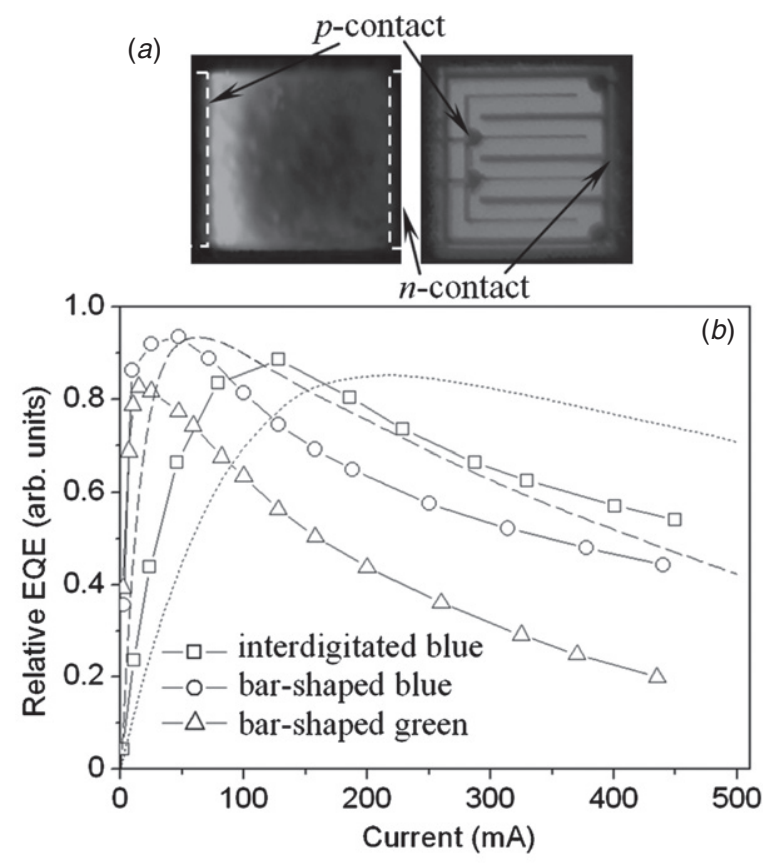

Figure 3. (a) The spatial view of the light emitted by blue LEDs with two different contact patterns. (b) Measured EQE versus current dependences for blue and green LEDs with conventional bar-shaped and interdigitated contact pattern. The dashed (bar-shaped structure) and dotted (interdigitated structure) lines represent the corresponding calculated dependences for blue LEDs.

(curves 3 and 4). Curve 1 represents the IQE of the same LED but without taking into account the nonuniform current density distributions (CC-free LED). This curve was calculated directly from equations (1) and (4) where current density $(J)$ was considered to be spatially independent (one-dimensional calculation). The CC-free LED shows no efficiency rolloff even at high current. The efficiency versus current dependence gradually flattens out at a current of $300 \mathrm{~mA}$. This result is consistent with the earlier reported one-dimensional calculations [16, 17] and photoluminescence measurements $[5,6]$ where it was suggested that Auger recombination alone is not sufficient to explain the efficiency droop in InGaN LEDs. However, the CC effect may be neglected in LEDs with patterned contacts and virtually no resistance of cladding layers. In real devices, this is not the case and thus the local current (carrier) density in the vicinity of the contacts may be much higher than the average current density used in the calculation of the CC-free LEDs efficiency or injected carrier density in photoluminescence measurements. For example, in a LED with narrow p-contact at $I=100 \mathrm{~mA}$ (average current density of $\sim 11 \mathrm{~A} \mathrm{~cm}^{-2}$ ), the peak current density $10^{2} \mathrm{~A} \mathrm{~cm}^{-2}$ corresponds to the injected carrier concentration of $\sim 10^{19} \mathrm{~cm}^{-3}$. At such a carrier concentration, the Auger recombination rate becomes higher than SRH and radiative recombination rates which results in a decrease of the total IQE as current increases. In a LED with wide p-contact, the current density is distributed more evenly and the total IQE starts to drop at higher current $(I>200 \mathrm{~mA})$.

Figure 3 shows the modification of the spatial distribution of light emitted by the blue LEDs with two different 
electrode patterns. In the case of interdigitated geometry, the electroluminescence is uniformly distributed over the whole emitting region which indicates the uniform current spreading in the active layer. Alternatively, in the case of conventional bar-shaped geometry, the light spatial distribution becomes remarkably nonuniform, even at a low injection level $(I>$ $1 \mathrm{~mA})$. The LED mostly emits light in the region close to the p-contact, thus indicating strong current line localization near the p-contact. The relative EQE versus current dependences for the LED samples with bar-shaped and interdigitated geometries are significantly different. In the case of bar-shaped geometry, the EQE reaches its peak at $I_{\text {peak }}=50 \mathrm{~mA}$. When interdigitated geometry is used, the efficiency roll-off current increases up to $I_{\text {peak }}=130 \mathrm{~mA}$. Moreover, the degree of the efficiency roll-off at high currents (defined as $\left(\mathrm{EQE}\left(I_{\text {peak }}\right)-\right.$ $\left.\left.\operatorname{EQE}\left(I_{\max }\right)\right) / \operatorname{EQE}\left(I_{\text {peak }}\right)\right)$ is $53 \%$ and $36 \%$ for bar-shaped and interdigitated LEDs respectively. Due to the fact that both LEDs are fabricated from the same wafer and all transport and recombination properties should be similar for both devices, the lower EQE of the bar-shaped LED is related to the strong $\mathrm{CC}$ in the active region. The data obtained are consistent with the numerical calculations presented above. However, the experimental values of efficiency droop are higher than those predicted theoretically. To explain this discrepancy, some issues should be considered. First, for typical MQW InGaN LEDs, the recombination occurs mainly in the QW that lies closer to the p-layers [18]. Moreover, the 'effective' thickness of the QW is less than the real thickness due to internal polarization fields which leads to spatial separation of electron and hole wavefunctions. Therefore, the injected carrier concentration in our calculations is underestimated. This leads to lower Auger recombination rate and overestimated LEDs efficiency as compared to experiment. Second, the total thirdorder nonradiative coefficient $(C)$ is found to be higher than $10^{-29} \mathrm{~cm}^{6} \mathrm{~s}^{-1}$ due to the contribution of carrier leakage out of the active region [19]. Besides, this value may be affected by the CC. The high internal electric field in the regions of current line localization may lead to lateral nonuniformity of the energy band diagram of the MQW structure and higher value of the leakage current as compared to the value predicted by one-dimensional calculations.

Next we consider the effect of CC on the efficiency of InGaN LEDs with different peak wavelengths (different In content in the QWs). It is known that the EQE rolloff decreases as the peak wavelength increases. Carrier delocalization from In-rich regions has been suggested to account for this effect [3]. In [20], it was shown that the wavelength-dependent efficiency droop is caused by several mechanisms including recombination-based mechanisms as well as transport-based mechanisms. We suggest that the $\mathrm{CC}$ may also be responsible for the higher droop effect in green-shifted LEDs. First, the CC is most pronounced in the longer wavelength LEDs, making less uniform lateral injection in these devices [21]. Second, when the In content increases, the injected hole concentration may be more nonuniform among the QWs due to higher quantum barrier in green-shifted LEDs [20]. Third, the calculation of the Auger recombination rate in InGaN alloys from the first principles predicts increasing of Auger coefficient with the In content in alloys [22]. The experimental investigations of the Auger coefficient dependence on the In content varying in wide range are less reported in the literature. Nevertheless, reference [14], reported on the Auger coefficient from $1.4 \times$ $10^{-30}$ to $2.0 \times 10^{-30} \mathrm{~cm}^{6} \mathrm{~s}^{-1}$ for the In content ranged from $9 \%$ (near-ultraviolet region) to $15 \%$ (blue region). Using linear extrapolation of these data, we estimate the Auger coefficient to be $3.4 \times 10^{-30} \mathrm{~cm}^{6} \mathrm{~s}^{-1}$ for In content $30 \%$ (green region). Figure 2 compares the calculated IQE versus current dependences for conventional bar-shaped blue and green LEDs. Similarly to the blue emitting devices, the CC-free green LED also shows negligible efficiency droop (curve 2). In contrast, the efficiency of the green device with nonuniform lateral injection (curve 5) reaches a peak value at lower current and demonstrates stronger droop as current increases, in comparison with the blue LED.

\section{Conclusions}

We have investigated the CC effect on the efficiency droop phenomenon in InGaN/GaN MQW LEDs on a sapphire substrate. We show that the efficiency droop is not uniquely determined by carrier recombination and transport mechanisms in the quantum well/barrier structure but also depends on the device design. The results of the experimental testing of lateral LEDs with different contact patterns indicate that the CC makes the efficiency droop to increase and roll-off current value to decrease. Simulations reveal that enhancement of the efficiency droop in LEDs with nonuniform current spreading originates from the $\mathrm{CC}$-affected increase of Auger recombination. Besides, the experimental data together with simulations suggest that the $\mathrm{CC}$ may be responsible for a higher droop effect in longer wavelength LEDs. In our opinion, the ways to minimize the effect of $\mathrm{CC}$ and Auger recombination on the efficiency of visible InGaN/GaN LEDs are the reduction of injected carrier concentration in the active region (double heterostructure) and improvement of current spreading (vertical injection geometry). However, the improvement process is limited due to (1) a trade-off between the CC effect and the light extraction efficiency and (2) a tradeoff between achieving high EQE and low efficiency droop.

\section{References}

[1] Gardner N F, Müller G O, Shen Y C, Chen G, Watanabe S, Götz W and Krames M R 2007 Appl. Phys. Lett. 91243506

[2] Zhang M, Bhattacharya P, Singh J and Hinckley J 2009 Appl. Phys. Lett. 95201108

[3] Cao X A, Yang Y and Guo H 2008 J. Appl. Phys. 104093108

[4] Chichibu S F, Azuhata T, Sugiyama M, Kitamura T, Ishida Y, Okumurac H, Nakanishi H, Sota T and Mukai T 2001 J. Vac. Sci. Technol. B 192177

[5] Xie J, Ni X, Fan Q, Shimada R, Özgür Ü and Morkoç H 2008 Appl. Phys. Lett. 93121107

[6] Kim M H, Schubert M F, Dai Q, Kim J K, Schubert E F, Piprek J and Park Y 2007 Appl. Phys. Lett. 91183507

[7] Ling S C, Lu T C, Chang S P, Chen J R, Kuo H C and Wang S C 2010 Appl. Phys. Lett. 96231101

[8] Zhao H, Liu G, Arif R A and Tansu N 2010 Solid-State Electron. 541119 
[9] Han S H, Lee D Y, Lee S J, Cho C Y, Kwon M K, Lee S P, Noh D Y, Kim D J, Kim Y C and Park S J 2009 Appl. Phys. Lett. 94231123

[10] Guo X and Schubert E F 2001 J. Appl. Phys. 904191

[11] Shatalov M et al 2002 Japan. J. Appl. Phys. 415083

[12] Schubert E F 2006 Light-Emitting Diodes 2nd edn (Cambridge: Cambridge University Press)

[13] Dai Q et al 2009 Appl. Phys. Lett. 94111109

[14] Shen Y C, Mueller G O, Watanabe S, Gardner N F, Munkholm A and Krames M R 2007 Appl. Phys. Lett. 91141101

[15] David A and Grundmann M J 2010 Appl. Phys. Lett. 96103504

[16] Yen S H, Tsai M C, Tsai M L, Shen Y J, Hsu T C and Kuo Y K 2009 Appl. Phys. A 97705
[17] Ryu H Y, Kim H S and Shim J I 2009 Appl. Phys. Lett. 95081114

[18] David A, Grundmann M J, Kaeding J F, Gardner N F, Mihopoulos T G and Krames M R 2008 Appl. Phys. Lett. 92053502

[19] Dai Q, Shan Q, Wang J, Chhajed S, Cho J, Schubert E F, Crawford M H, Koleske D D, Kim M H and Park Y 2010 Appl. Phys. Lett. 97133507

[20] Chen J R, Wu Y C, Ling S C, Ko T S, Lu T C, Kuo H C, Kuo Y K and Wang S C 2010 Appl. Phys. B 98779

[21] Malyutenko V K, Zinovchuk A V and Malyutenko O Y 2008 Semicond. Sci. Technol. 23085004

[22] Delaney K T, Rinke P and Van de Walle C G 2009 Appl. Phys. Lett. 94191109 